\title{
Dengue Fever: A Challenge to Health System
}

\author{
Ghulam Murtaza Gondal \\ Professor of Medicine, Foundation University Medical College, Rawalpindi
}

Dengue fever is considered one of the most threatening mosquito transmitted disease with 30 -fold rise in incidence world-wide. Since the first ever known outbreak of dengue infection in Pakistan reported in 1994, there are increasing cases of variable intensity every year. In the current year, Pakistan is undergoing one of its worstever dengue fever epidemics. The first case was reported on $8^{\text {th }}$ July 2019 in Peshawar and since then it has spread like a fire all over the country apart from Islamabad, capital of Pakistan. From July to November 2019, a total of 47,120 confirmed patients having this disease and 175 deaths are being notified ${ }^{1}$.

The disease has hit urban areas of Pakistan, placing hospitals under severe strain. Authorities are conducting anti-mosquito spraying in urban areas to contain the spread of disease butdue to limited resources, prolonged monsoon rains, lack of anticipation and timely measures by health authorities led to the outbreak. ${ }^{1}$

Dengue fever is a viral infection caused by virus belonging to the Flaviviridae family. It is transmitted by bite of an infected female Aedes aegypti mosquitoes that feed during daytime (most cases at Fajr and Maghreb prayer time). ${ }^{2}$ This mosquito multiplies at places where there is plenty of clean stagnant water like ponds, underwater tank, jars, some empty container and old used tyres. Lack of knowledge regarding disease prevention, proper sanitation and regular garbage collection is also contributing to the spread of the mosquitoes in urban as well as rural areas. ${ }^{3}$ Risk of Dengue exists worldwide in tropical and subtropical areas like Central and South

\section{Correspondence:}

Ghulam Murtaza Gondal

Email:drgmgondal@gmail.com

Cite this Editorial: Gondal GM. Dengue Fever: A challenge to Health System. J Islamabad Med Dental Coll. 2019;8(4):163-165. Doi: 10.35787/jimdc.v8i4.474
America, African countries and Asia especially in the subcontinent, including Pakistan. Therefore, all people travelling to affected areas should be considered at high risk for disease acquisition during outbreaks. ${ }^{4}$

The initial symptoms are mild body aches, fever with runny nose and itching go unnoticed and are usually not investigated. Those who develop illness usually develop symptoms during the first week after the bite of infectious mosquito. Early infection presents with flu-like symptoms (high-grade fever, redness and painful reddened eyes, severe myalgia, bone and joint pain, intractable headache), itching all over body along with a diffuse petechial or erythematous rash on skin. ${ }^{5}$ If untreated the illness occasionally may progress to dengue hemorrhagic fever (DHF ), in which patients present with pain in abdomen, loose stools, fits, pleural effusion, ascites and excessive tendency to bleed. Further complications may result in circulatory failure, shock and death (also known as Dengue Shock Syndrome). An important fact to recognize is that if a patient gets infected by same serotype of Dengue virus, it results in some immunity to future attacks of the virus. However, if the patient gets infected by another serotype, there is more chance to develop dengue hemorrhagic fever. Therefore, once dengue infection occurs, it is very important to prevent patients from further attacks in same as well as in subsequentseasons. ${ }^{6}$

Laboratory confirmation of dengue fever involves isolation of virus, nucleic acid of virus, its antigen, antibody to dengue virus, or any combination of these diagnostic methods. In any suspected patient of dengue fever, organism can be isolated from serum, circulating RBCs or tissues for about 5 days by dengue NS1 antigen. ${ }^{7}$ After the acute phase of disease, serological tests like lgM and $\lg \mathrm{G}$ antibodies in serum are considered as diagnostic tests for dengue infection. ${ }^{8}$ Ultrasound done timely is al so a cost-effective and easily available diagnostic modality 
for evaluating cases of suspected Dengue Hemorrhagic fever. This technique detects pleural effusion, ascites and thickening of gall bladder wall, all of which represent significant permeability of blood vessels. ${ }^{9}$ The utility of ultrasound in previous studies was limited because patients underwent only a single scan. Currently, serial ultrasound examination of thorax and abdomen of patients with dengue fever has been found very helpful in detecting patients who are progressing to severe form of the disease..$^{10}$

So far, there is no proper therapy for mild or severe dengue fever, except supportive care along with methods for prevention. For severe aches, fever and pain Paracetamol can be used orally or in intravenous infusion. Patients are advised complete rest and plenty of fluids orally (IN fluids if no oral intake due to vomiting). Patients with worsening of symptoms in the first 24 hours should be admitted to hospital immediately to monitor for disease progression and complications. ${ }^{11}$

The only effective way of controlling this infection is protection from bite of infected mosquito, especially people travelling to endemic areas. Measures include protection of human beings from mosquito bites and taking measures for keeping control on mosquito population. Protective measures from dengue infection include use of anti-mosquito lotions, wearing long shirts and long pants which should be tucked into socks while going outside. The mosquito population can be reduced by getting rid of places where mosquitoes can breed e.g. used tyres, water cans, or household plant pots where rain water can collect, outdoor bird and pets' water dishes.12 Recently FDA has approved a vaccine, Dengvaxia, which is claimed to help in prevention of Dengue fever in children aged 9 to 16 years who are already infected by the Dengue virus. ${ }^{13}$ However, it is not available worldwide to prevent the disease in the general population, especially in endemic and under developed countries.

Given the fact that dengue fever is a challenge to the existing health system and a burden on health services, it could be tackled by applying simple, correct and timely treatment strategies. The management of dengue demands multi-pronged approach, involving relevant stakeholders beyond health sector and effectively implementing preventive strategies with cost-effective interventions. Dengue is becoming a global threat and it is a need of time to adopt worldwide strategy for its prevention and treatment by promoting integrated approach of vector management and sustainable control measures at all levels to reduce morbidity and mortality from dengue fever.

\section{References}

1. World Health Organization press release on outbreak of Dengue infection in Pakistan. 2019

2. Rasheed SB, Butlin RK, Boots M. A review of dengue as an emerging disease in Pakistan. Public Health. 2013; 127(1): 11-7. Doi: 10.1016/j.puhe.2012.09.006

3. Bostan N, Javed S, Nabgha EA, Eqani SA, Tahir $F$, Bokhari $H$. Dengue fever virus in Pakistan: effects of seasonal pattern and temperature change on distribution of vector and virus. Rev Med Virol. 2017; 27(1): 112-7. Doi: 10.1002/rmv.1899

4. Heydari M, Metanat M, Rouzbeh-Far MA, Tabatabaei SM, Rakhshani M, Jahromi M. Dengue Fever as an Emerging Infection in Southeast Iran, Am J Trop Med Hyg. 2018: 98(5):1469-71. Doi: 10.4269/ajtmh.17-0634

5. Ahmad Qureshi EM, Tabinda $A B$, Vehra $S$. Predicting dengue outbreak in the metropolitan city Lahore, Pakistan, using dengue vector indices and selected climatological variables as predictors. J Pak Med Assoc. 2017; 67(3): 41621. PMID: 28303992

6. Bhatt S, Gething PW, Brady OJ, Messina JP, Farlow AW, Moyes $\mathrm{CL}$, et al. The global distribution and burden of dengue. Nature. 2013; 496(7446): 504-7. Doi:10.1038/nature12060

7. Nascimento EJM, George JK, Velasco M. Development of an anti-dengue NS1 IgG ELISA to evaluate exposure to dengue virus. J Virol Methods. 2018; 257-8. Doi: 10.1016/j.jviromet.2018.03.007

8. Wilson ME, Chen LH. Dengue: update on epidemiology. Curr Infect Dis Rep. 2015: 17 (1): 457-61. Doi: 10.1007/s11908-014-0457-2

9. Chatterjee R, Mysore A, Ahya K. Utility of sonography in clinically suspected Dengue. Pediatr Infect Dis. 2012; 4(3): 107-11. Doi: 10.1016/j.pid.2012.07.006

10. Michels $M$, Sumardi $U$, de Mast $Q$, Jusuf $H$, Puspita M. The Predictive Diagnostic Value of Serial Daily Bedside Ultrasonography for Severe 
Dengue in Indonesian Adults. PLOS Negl. Trop. Dis. 2013: 7(6): e2277. Doi: 10.1371/journal.pntd.0002277

11. Rajapakse S, de Silva NL, Weeratunga P. Prophylactic and therapeutic interventions for bleeding in dengue: a systematic review. Trans R Soc Trop Med Hyg. 2017; 111(10):433-7. Doi: 10.1093/trstmh/trx079
12. WHO. Dengue: guidelines for diagnosis, treatment, prevention and control - new edition. WHO, Geneva. 2009

13. World Health Organization. Weekly epidemiological record. Dengue vaccine: WHO position paper.

2018. 